\title{
Flexural Capacity Calculation Approach for Precast Grouted Shear Wall Influenced by Joint Interface Displacements
}

\author{
Dong-yue Wu, ${ }^{1}$ Shu-ting Liang, ${ }^{1}$ Zheng-xing Guo, ${ }^{1}$ and Xiao-jun $\mathrm{Zhu}^{2}$ \\ ${ }^{1}$ College of Civil Engineering, Southeast University, Nanjing 210096, China \\ ${ }^{2}$ Architects and Engineering Co., Ltd., Southeast University, Nanjing 210096, China \\ Correspondence should be addressed to Shu-ting Liang; stliang@seu.edu.cn
}

Received 30 April 2015; Revised 18 August 2015; Accepted 19 August 2015

Academic Editor: Antônio G. B. de Lima

Copyright (C) 2015 Dong-yue Wu et al. This is an open access article distributed under the Creative Commons Attribution License, which permits unrestricted use, distribution, and reproduction in any medium, provided the original work is properly cited.

\begin{abstract}
The grouted precast buildings have great economic superiorities and application potentials. With excessively utilized confinement steels, connecting steels, and steel sleeves, their mechanical properties satisfied the strength requirements. But the capacity calculation approach still remains uncertain due to the complexity caused by the joint interface slip and joint gap opening displacement, which can hardly be avoided and lead to an excessive steel configuration. In order to make the design approach of precast grouted shear wall convenient and to achieve economic superiorities and properties advances, this paper proposed a new flexural capacity calculation approach considering joint interface displacements influences and properties improvement by confinement steels. The calculated capacities of example precast shear wall are $553.8 \mathrm{kN}, 501.0 \mathrm{kN}$, and $536.4 \mathrm{kN}$ when only considering confinement improvement, interface slip reduction, and both the improvement and reduction, respectively. By comparing the calculated results with the experimental tested results of $589.4 \mathrm{kN}$, the accuracy and reasonability of this new calculation approach were verified. Afterward, some requirements on application conditions of this new calculation approach were suggested depending on different precast structure type and seismic area.
\end{abstract}

\section{Introduction}

The industrialization and standardization are the basic characteristics of modern civil engineering; as a result, precast structure became an irreversible developing trend. The shear wall structure, whose capacity and lateral stiffness are both high, is an ideal lateral force resisting structure in highrise building. So, combining the precast concrete technology with the shear wall structure, to adapt the industrialization and standardization requirements, the precast grouted shear wall structure has been developed and researched by many scholars. This kind of precast structure is characterized by the grouted connected steel bars as the core factor relating to the structure properties.

Overall, the precast shear wall connecting joints can be divided into two different types based on their deformation properties: the flexible connecting joints and the strong connecting joints $[1,2]$. The flexible connecting joints allow larger lateral and gap opening displacements and utilize these larger displacements cooperating with dampers to dissipate earthquake energy, such as the hybrid unbonded posttension precast shear wall developed by Kurama in the PRESSS research program $[3,4]$. On the other hand, the strong connecting joints require that the mechanical properties of precast joint should be not weaker than that of the castin-situ joint, meaning that the joint deformation should be limited. Some early researches indicated that both the flexible and strong connecting joints expressed good mechanical properties, while the application fields of the two connectors are different. Taking the hybrid unboned posttension precast shear wall as an example, which was firstly developed in the PRESSS program in 1990's and by further improvements expresses good energy dissipation and repair ability, due to its large deformation, it is not suitable in high-rise precast buildings because large lateral displacement will cause large second-order moment. On the contrast with the flexible connecting joints, the strong connecting joints' application field is wide, but the mechanical requirements are stricter and the expenses are higher. So, in the precast high-rise shear wall structures, the strong connecting joints will be more suitable. 


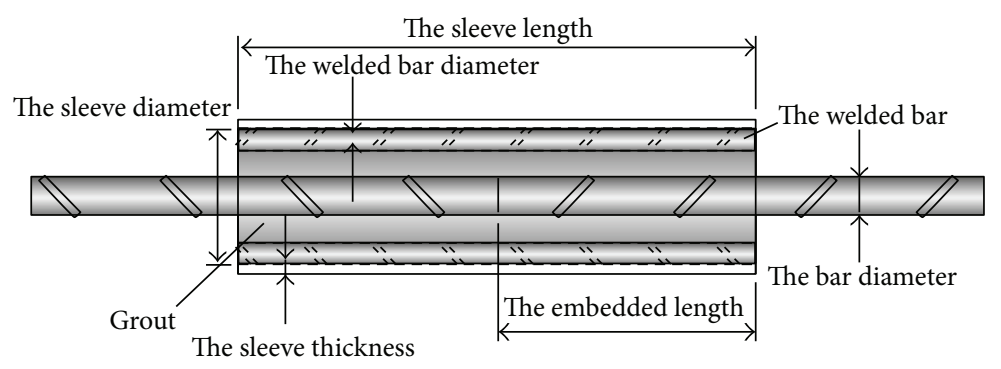

FIGURE 1: A typical grouting connected steel bar using steel sleeve.

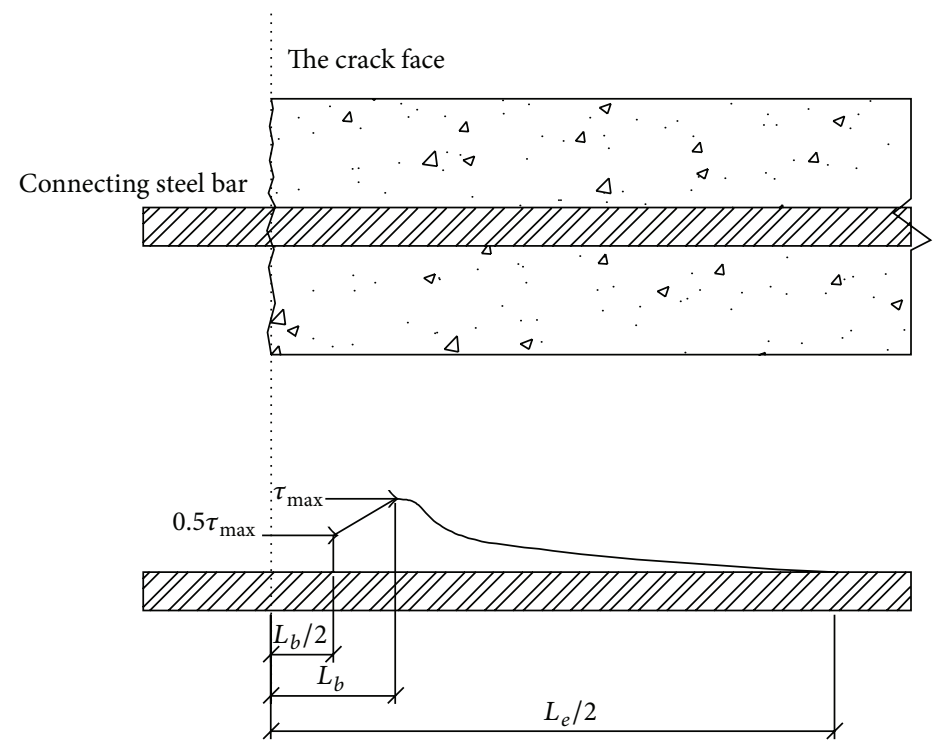

FIGURE 2: The bond shear stress distribution on connecting steel bar surface under gap opening displacement.

The steel bar grouted precast shear wall joint is a typical strong connecting joint.

Ever since the first invention of steel grouting connector by Yee in the late 1960s [5], numerous researches had been carried out to develop new effective connector and various types of steel grouting connectors have been invented subsequently. Figure 1 shows one typical grouting connected steel bar using steel sleeve [6]. While even utilizing steel sleeves can realize the connecting mechanical properties not less than that of the intact steel bar, one vital factor cannot be ignored, which has dramatic effects on the joint properties: the precast joint interface displacements. Some of the experimental test of the precast shear wall carried out by Smith et al. [2], Jiabin et al. [7], and Kang et al. [8] indicated that even in strong connecting joint the joint interface slip and the gap opening displacement hardly can be avoided, especially for the precast shear wall with low axial force. Till now, most of the precast strong connecting joints utilized an enlarged amount of connecting steels to reduce the harmful effects of the joint interface slip and gap opening. But, how many amounts of steels should be enlarged still remains uncertain and sometimes depends on experiences. So understanding the effect law of joint interface slip and gap opening displacement to connecting steel bars and proposing an accurate and reliable capacity calculation approach for precast shear wall connecting joints will be very critical in the future precast shear wall research.

\section{The Effect of the Joint Displacements on the Steel Stresses}

2.1. Simplifications on the Precast Joint Interface Interactions. In precast shear walls, precast joint interface can be considered as an original crack crossing the whole precast shear wall section. And on this original crack, the stress distribution of connecting bars and the interactions are complex. Soltani et al. [9] concluded the interactions of the cast-in-situ crack that the reinforced concrete panel is modeled as a multicomponent structural system composed of reinforcing bars and concrete as well as their interaction. Structural nonlinearity and local stress field comprise deferent size dependent stress transfer mechanisms and phenomena. Figure 2 is the bond shear stress distribution on connecting steel bar surface caused by gap opening displacement and Figure 3 is the bending of connecting steel bar caused by joint interface slip. But, 


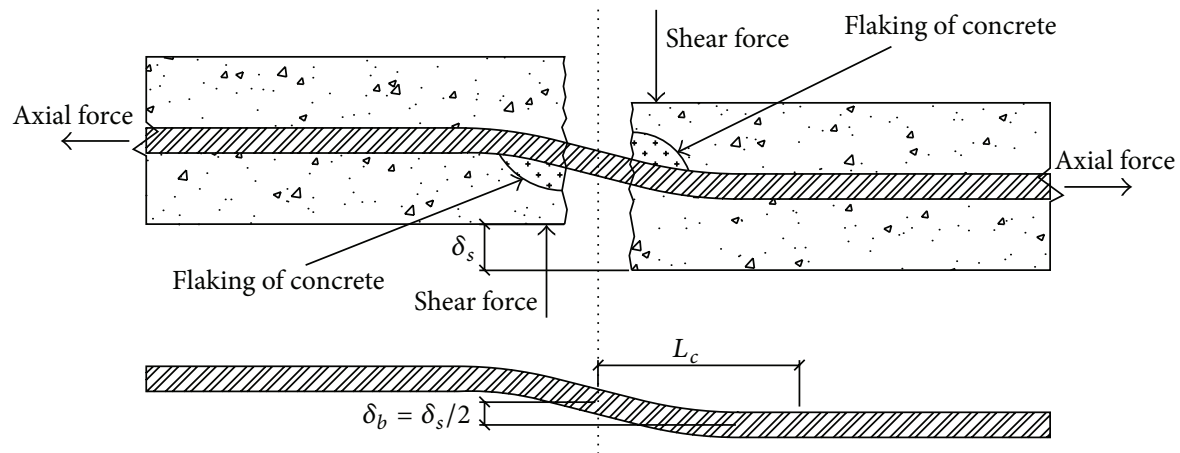

FIGURE 3: The bending of connecting steel bar under joint interface slip.

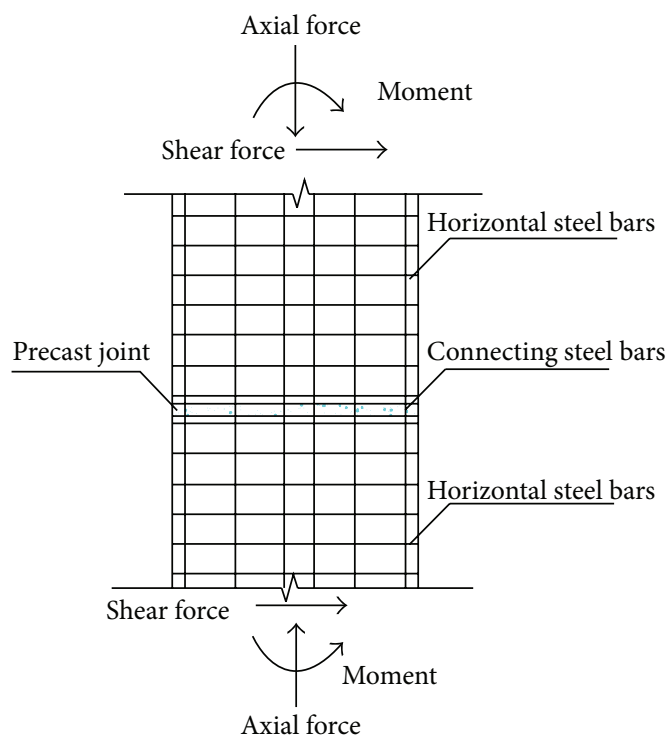

(a) Connecting steel bar group crossing joint

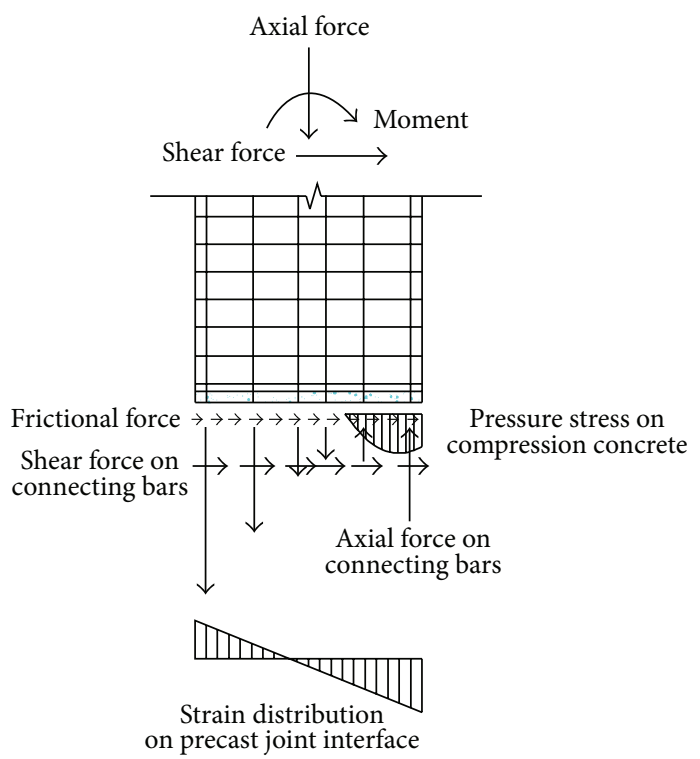

(b) Forces and strain distribution of connecting steel bars

FIGURE 4: Connecting steel bar group crossing precast shear wall joint and forces and strain distribution.

in precast shear wall joints, the "original crack" is a special crack whose surface is straight and the bonded strength is very low comparing to frictional force and the stress on connecting steel bars. So, the stress transfer mechanisms can be simplified as the following: (1) dowel action and kinking of connecting steel bars at the joint interface location, (2) frictional force related with the axial force and carried by the concrete interface.

\subsection{The Relationship of Gap Opening Displacement and the} Axial Stress. Ling et al. [10] studied the relationship of joint gap opening displacement and the axial force on a single connecting steel bar with steel sleeve and concluded that the preyield and postyield load-displacement behaviors of the grouted splice are expressed as the following when the gap opening displacement ranges are $0 \leq \delta_{v}<2.33(\mathrm{~mm})$ and $\delta_{v}>2.33(\mathrm{~mm})$, respectively:

$$
\begin{aligned}
& P=47.1 \delta_{v} \quad 0 \leq \delta_{v}<2.33, \\
& P=-0.0484 \delta_{v}^{2}+2.45 \delta_{v}+104 \quad \delta_{v} \geq 2.33,
\end{aligned}
$$

where $P$ is the tension load $(\mathrm{N})$ and $\delta_{v}$ is the displacement of the spliced bar $(\mathrm{mm})$, which equals the interface gap opening displacement on the steel bar location.

For the connecting steel bar group locating on precast shear wall joint, the strain distribution on different connecting steel bars satisfied the plane section assumption as shown in Figure 4.

2.3. The Relationship of the Joint Interface Slip and the Dowel Action Shear Stress. The joint interface slip will cause the bend and dowel action on the connecting steel bars. The connecting bars under coupled axial load and transverse displacement are bent in vicinity of the interface. Due to localization of curvature in the connecting steels close to the joint interface, the axial stiffness and the main yield strength of the connecting steels are reduced. In order to consider this effect, the curvature-influencing zone close to the precast joint interface $L_{c}$ is considered. Based on the experiments, the curvature-influencing zone was observed to be between $4 d_{s}$ and $5 d_{s}$ initially with a small increase in large displacement. 
In small displacement when both the connecting bar and concrete are in elastic range, the size of curvature zone can be derived from modeling of bars as a beam resting on an elastic foundation as [9]

$$
\begin{aligned}
L_{c 0} & =\frac{3 \pi}{4} \sqrt[4]{\frac{4 E_{s} I_{s}}{K d_{s}}}, \\
K & \left.=\frac{150 f_{c}^{\prime 0.85}}{d_{s}} \text { (Unit: } \mathrm{MPa} / \mathrm{mm}\right) .
\end{aligned}
$$

In large deflection, the connecting bars and supporting concrete show nonlinear behavior and consequently the curvature zone increases. This effect will be considered by proposing an empirical nondimensional damage parameter as follows:

$$
\mu=\left(1+150 \frac{\delta_{v}}{d_{s}}\right) \frac{\delta_{b}}{d_{s}} .
$$

Finally, the curvature zone is expressed as follows:

$$
L_{c}=\frac{3 \pi}{4} \sqrt[4]{\frac{4 E_{s} I_{s}}{K d_{s}}}\left(1+150 \frac{\delta_{v}}{d_{s}}\right) \frac{\delta_{s}}{2 d_{s}} .
$$

By the experimental test [9], the shape of curvature distribution $\phi(x)$, within $L_{c}$, can be mathematically modeled by a skew parabolic form as follows:

$$
\begin{aligned}
& \phi(x)=\frac{3 \phi_{\max }}{L_{c}^{2}}\left[x-\left(\frac{L_{e}}{2}-L_{c}\right)\right]^{2}, \\
& \frac{L_{e}}{2}-L_{c} \leq x<\frac{L_{e}}{2}-\frac{L_{c}}{2}, \\
& \phi(x)=\frac{-3 \phi_{\max }}{L_{c}^{2}}\left\{3\left[x-\left(\frac{L_{e}}{2}-\frac{L_{c}}{2}\right)\right]^{2}\right. \\
& \left.-L_{c}\left[x-\left(\frac{L_{e}}{2}-\frac{3 L_{c}}{4}\right)\right]\right\} \quad \frac{L_{e}}{2}-\frac{L_{c}}{2} \leq x<\frac{L_{e}}{2},
\end{aligned}
$$

where the maximum curvatures can be calculated to satisfy the compatibility condition as

$$
\delta_{s}=2 \iint_{L_{e} / 2} \phi(x) d x .
$$

Therefore, the maximum curvatures are as follows:

$$
\phi_{\max }=\frac{32 \delta_{s}}{11 L_{c}^{2}} .
$$

Based on assumed distribution profiles of bond stress and curvature along the curvature zone (5), the sectional averaged mean stress and strain along this region can be computed. The strain for each fiber of cross section is calculated as follows:

$$
\varepsilon_{f}=\varepsilon_{s}(x)+\phi(x) y,
$$

where $y$ is the local coordinate of steel fiber measured from the center of the bar cross section, $\varepsilon_{s}(x)$ is the averaged tensile strain of the section, and $\varepsilon_{f}$ is the fiber strain of connecting steel bar. For any fiber strain, the stress will be obtained from the uniaxial stress-strain relationship of bare steel bar. Therefore, the internal cross section force, including the bending moment $M(x)$ and shear force $V(x)$, along the steel bar axis, is also computed as follows:

$$
\begin{aligned}
& M(x)=\int_{-d / 2}^{d / 2} \sigma_{f}(\phi(x), y) y d A_{s}(y) \\
& V(x)=\frac{d M(x)}{d x}
\end{aligned}
$$

Therefore, the shear stress carried by connecting bar, socalled "dowel action shear stress," is directly calculated:

$$
\tau(x)=\frac{V(x)}{A_{s}} .
$$

2.4. Parametric Study. The precast joint can be treated as a special straight original crack, whose interface slip will cause the dowel action shear stress in the connecting steel bars, while this special crack also has its special characteristics differing from a cast-in-situ crack. (1) The interface slip can be considered as equal at any place in the compression region of precast joint section; (2) the gap opening displacement only exists in the tension region of the joint interface section and the gap opening displacement in the compression region remains zero; (3) for the gap opening displacement in the tension region, the gap opening displacement distribution is a liner plane section distribution starting from the natural axis.

Assuming that the stress-strain relationship of steel bars is the elastic-plastic relationship:

$$
\begin{aligned}
& \text { Elastic stage: } \sigma_{s}=E_{s} \varepsilon_{s} . \\
& \text { Plastic stage: } \sigma_{s}=f_{y} .
\end{aligned}
$$

The maximum shear stress in the connecting steel bar can be obtained as follows:

$$
\begin{aligned}
\tau & =\frac{4 E_{s} \xi_{h}}{11\left((3 \pi \eta / 4) \sqrt[4]{\pi E_{s} / 2400 f_{c}^{0.85}}\right)^{3}}, \\
\eta & =1 \\
\xi_{h}+150 \xi_{h} \xi_{v} & <0.02 \\
\eta & =1+3\left(\xi_{h}+150 \xi_{h} \xi_{v}-0.02\right)^{0.85} \\
\xi_{h}+150 \xi_{h} \xi_{v} & \geq 0.02
\end{aligned}
$$

where $\xi_{s}$ and $\xi_{v}$ are two nondimensional parameters considered to eliminate the effect of steel bar diameter. $\xi_{s}$ is the interface slip-diameter ratio and $\xi_{v}$ is the vertical gap opening displacement-diameter ratio:

$$
\begin{aligned}
& \xi_{s}=\frac{\delta_{s}}{d_{s}}, \\
& \xi_{v}=\frac{\delta_{v}}{d_{s}} .
\end{aligned}
$$




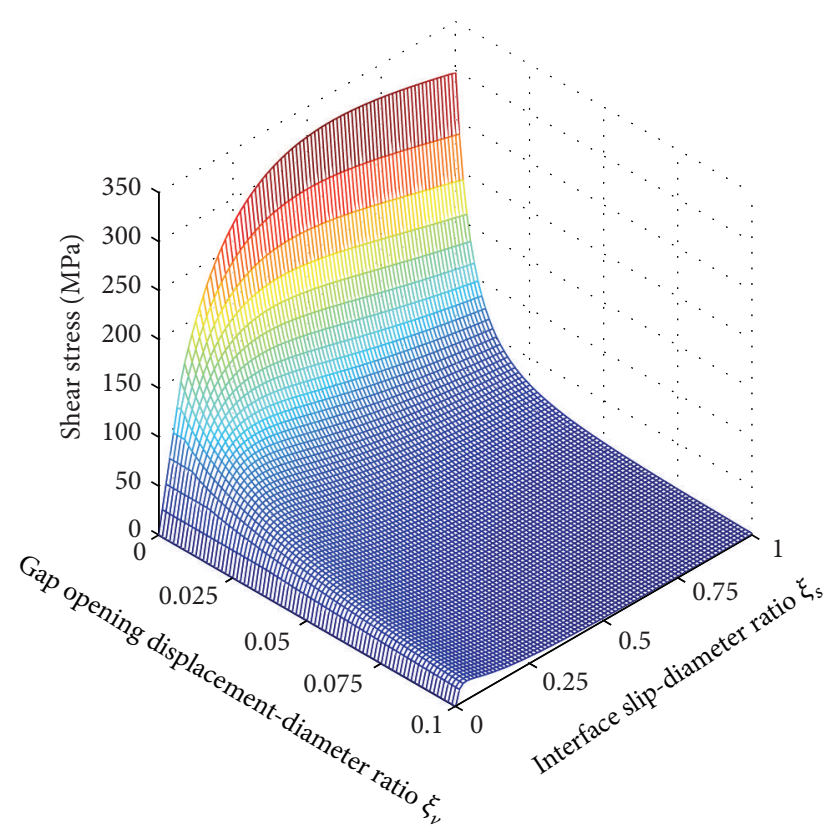

FIGURE 5: The space curved surface of $\xi_{s}$ and $\xi_{v}$ to shear stress of single steel bar.

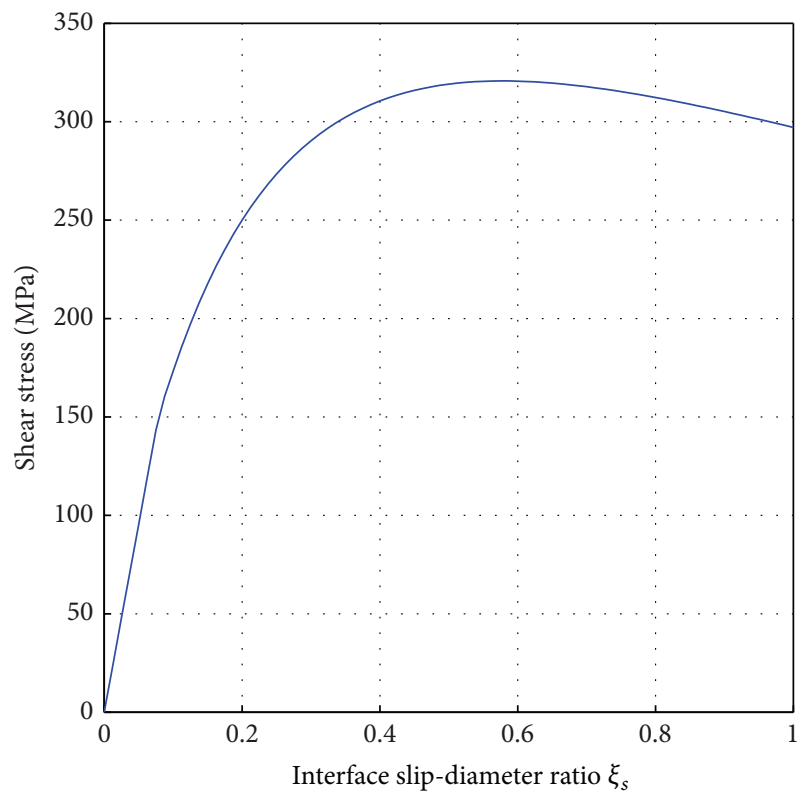

Figure 6: The curve of $\xi_{s}$ to shear stress of single steel bar in compression zone.

Taking the HRB 400 steel as a calculating example, the yield stress $f_{y}$ is $400 \mathrm{MPa}$ and the elastic modulus $E_{s}$ is $200 \mathrm{GPa}$. The calculated results are presented in Figures 5-8.

Figure 5 is the space curved surface of the lateral shear stress, the gap opening displacement-diameter ratio, and the lateral joint interface slip-diameter ratio. The space curved surface indicates that both the gap opening displacement and the joint interface slip will influence the lateral shear stress of steel bar dramatically, while the influence of the two

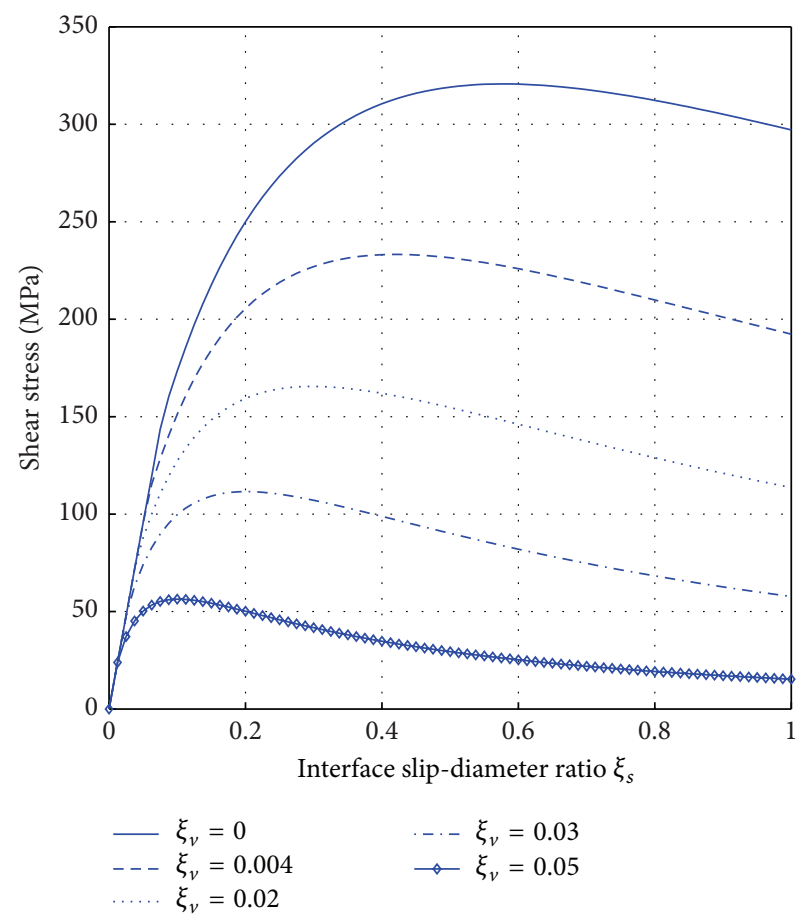

FIGURE 7: The curve of $\xi_{s}$ to shear stress of single steel bar with different $\xi_{v}$.

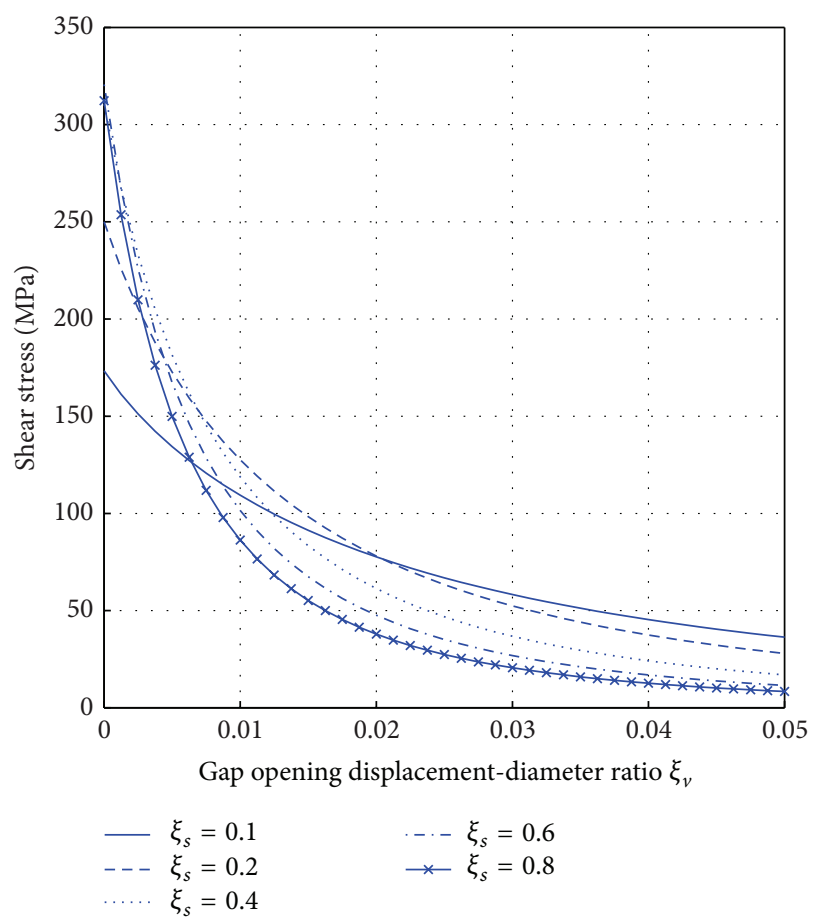

Figure 8: The curve of $\xi_{v}$ to shear stress with different $\xi_{s}$.

parameters varies in trend and amount. For the gap opening displacement, the dowel action shear stress will decrease with the gap opening displacement-diameter ratio increasing. On the contrast, the interface slip's increasing will firstly increase the dowel action shear stress and then decline it. The gap opening displacement-diameter ratio influences the dowel 
action shear stress more dramatically than the interface slipdiameter ratio, indicating that, in tension region of precast shear wall joint section, the dowel action shear stress may be very low due to a larger gap opening displacement.

Figure 6 is the curve of the steel bar shear stress and lateral interface slip-diameter ratio when the gap opening displacement is zero. Also, this illustrates the relationship between steel bar shear stress and interface slip-diameter ratio in compression region. The curve in Figure 4 indicated that the steel bar shear stress will increase with the interface slip-diameter ratio increase and then decrease after the interface slip-diameter ratio reached 0.575 . The maximum shear stress is $320.7 \mathrm{MPa}$, which is a very high value and $80.2 \%$ of the yield stress (400 MPa). But usually, the maximum shear stress is difficult to be reached because the 0.575 interface slipdiameter ratio means a very large interface slip; for example, it will be a $4.6 \mathrm{~mm}$ interface slip for steel bar with diameter of $8 \mathrm{~mm}$.

Figure 7 is the curve of the lateral interface slip-diameter ratio to the steel bar shear stress with different gap opening displacement-diameter ratios. It indicated that, with the gap opening displacement-diameter ratio increasing, the maximum shear stress and the related interface slip-diameter ratio decrease, but the development trend of the curve is similar to the curve in Figure 6, increases, and then decreases with the increasing of interface slip-diameter ratio. Figure 8 is the curves of the shear stress to the gap opening displacementdiameter ratio when the interface slip-diameter ratios are 0.1 , $0.2,0.4,0.6$, and 0.8 , respectively. Both curves in Figures 7 and 8 show that the gap opening displacement-diameter ratio influences the steel bar shear stress more dramatically than interface slip-diameter ratios.

\section{Flexural Capacity Calculation Equations for Precast Grouted Shear Wall}

3.1. The Calculation Model for Precast Grouted Shear Wall. Figure 4 is the simplified precast grouting shear wall calculation model. This model adopted the following assumptions:

(1) No matter what kind of steel bar connector is utilized, the connector can completely achieve the connection of the steel bars with the upper or lower precast concrete elements. The failure of the steel bar connector will be neglected in order to focus on the influences of joint interface displacements on the joint interface mechanical properties.

(2) Assume that the concrete strain distribution on the joint interface is the plant section distribution as shown in Figure 4.

(3) Neglect the grouted bond force on joint interface and the tension capacity of concrete.

(4) Neglect the dowel shear stress of connecting steel bars in tension region. For the connecting steel bars in the compression region, the lateral shear force acting on the precast wall is carried by the concrete interface frictional force and connecting steel bar dowel action. Therefore, because the interface slip in any compression position of the precast joint section is a constant, the dowel action shear stress of steel bars will be as follows:

$$
\tau=\frac{\left(V-\mu_{f} N\right)}{A_{s}} .
$$

3.2. The Calculation Equations for Cast-In-Situ Shear Walls. For the cast-in-situ shear wall, most of the design rules have given out the actual design approach [11, 12]. Chapter 9.4.3 of the "Code for Design of Concrete Structure" [11] gives out the calculation equations for cast-in-situ shear wall under lateral and axial loads: the design of cast-insitu shear wall should consider the eccentric compression or eccentric tension strength for the forward section and the diagonal shear strength. Chapters 6.2.17 and 6.2.19 give out the eccentric compression or eccentric tension strength calculation equations for rectangular, I shape, and $\mathrm{T}$ shape forward section of cast-in-situ shear wall with uniform steel bars in wall panel:

$$
\begin{aligned}
& N_{f} \\
& \quad \leq \alpha_{1} f_{c}\left[\xi b h_{0}+\left(b_{f}^{\prime}-b\right) h_{f}^{\prime}\right]+f_{y}^{\prime} A_{s}^{\prime}-\sigma_{s} A_{s}+N_{s w}, \\
& N_{f} e \\
& \leq \alpha_{1} f_{c}\left[\xi(1-0.5 \xi) b h_{0}^{2}+\left(b_{f}^{\prime}-b\right) h_{f}^{\prime}\left(h_{0}-\frac{h_{f}^{\prime}}{2}\right)\right] \\
& \quad+f_{y}^{\prime} A_{s}^{\prime}\left(h_{0}-a_{s}^{\prime}\right)+M_{s w}, \\
& e_{i}=\frac{e_{i}}{N_{f}}-\frac{h,}{N_{f}}+e_{a}, \\
& N_{s w}=\left(1+\frac{\xi-\beta_{1}}{0.5 \beta_{1} \omega}\right) f_{y w} A_{s w}, \\
& M_{s w}=\left[0.5-\left(\frac{\xi-\beta_{1}}{\beta_{1} \omega}\right)^{2}\right] f_{y w} A_{s w} h_{s w} .
\end{aligned}
$$

Chapter 6.3.21 gives out the diagonal shear strength calculation equation for the cast-in-situ shear wall under eccentric compression or eccentric tension loads:

$$
V_{f} \leq \frac{1}{\lambda-0.5}\left(0.5 f_{t} b h_{0}+0.13 N \frac{A_{w}}{A}\right)+f_{y v} \frac{A_{\mathrm{sh}}}{s_{v}} h_{0},
$$

where $N$ is the axial force acting on shear wall and when $N$ is larger than $0.2 f_{c} b h, N$ will be equal to $0.2 f_{c} b h$.

But for the precast shear wall, all the equations should be modified considering the characteristics of precast shear wall: the gap opening displacement and joint interface slip. Apart from that, there are usually more confinement steels or steel sleeves in the vicinity area of the joint comparing to cast-in-situ shear wall, leading to the concrete properties 
improved rapidly, concluded by Deng and Qu [13]. So, the improvement of the confinement steels to the concrete should also be considered.

3.3. The Constitutive Equations of Confined Concrete. Samani and Attard [14] proposed one of the most important stressstrain constitutive equations for confined concrete as the following:

Increasing stage is as follows:

$$
\frac{\sigma}{f_{0}}=\frac{A \cdot X+B \cdot X^{2}}{1+C \cdot X+D \cdot X^{2}}, \quad 0 \leq X \leq 1 .
$$

Descending stage is as follows:

$$
\begin{aligned}
\frac{\sigma}{f_{0}} & =\frac{f_{\text {residual }}}{f_{0}}+\left(1-\frac{f_{\text {residual }}}{f_{0}}\right)\left(\frac{f_{r}}{f_{c k}}\right)^{\left(\left(\varepsilon-\varepsilon_{0}\right) /\left(\varepsilon_{i}-\varepsilon_{0}\right)\right)^{2}} \\
X & =\frac{\varepsilon}{\varepsilon_{0}} \\
A & =\frac{E_{t i} \varepsilon_{0}}{f_{0}}=\frac{E_{c} \varepsilon_{0}}{f_{0}} \\
B & =\frac{(A-1)^{2}}{0.55}-1 \\
C & =A-2 \\
D & =B+1
\end{aligned}
$$

where $f_{0}$ is the maximum stress of confined concrete, which can be determined by (21). $\varepsilon_{0}$ is the strain at the maximum stress of confinement concrete, which can be determined by (22). For the secant modulus of concrete $E_{c}$, the secant modulus from zero stress point to $0.45 f_{0}$ stress point is usually taken. $f_{\text {residual }}$ is the residual strength of confined concrete, which can be determined by (23):

$$
\begin{aligned}
f_{0} & =f_{c k}\left(\frac{f_{r}}{f_{t k}}+1\right)^{1.25\left(f_{c k}\right)^{-0.21}\left(1+0.062\left(f_{r} / f_{c k}\right)\right)}, \\
\varepsilon_{0} & =\varepsilon_{c}\left[1+\left(17-0.006 f_{c k}\right)\left(\frac{f_{r}}{f_{c k}}\right)\right], \\
f_{\text {residual }} & =f_{0}\left[1-\frac{1}{\alpha\left(f_{r} / f_{c k}\right)^{k}+1}\right], \\
\alpha & =795.7-3.291 f_{c k}, \\
k & =\left[5.79\left(\frac{f_{r}}{f_{0}}\right)^{0.694}+1.301\right],
\end{aligned}
$$

$f_{r}$ is the confining stress acting on the concrete caused by confinement steel; the confining stress of rectangular section can be determined as follows:

$$
\begin{aligned}
& f_{r}=0.26\left[\frac{\rho_{s x}+\rho_{s y}}{s\left(b_{c x}+b_{c y}\right)}\right], \\
& \rho_{s x}=\sum A_{s x} f_{y t} \sqrt{\left(\frac{b_{c y}}{s}\right)\left(\frac{b_{c y}}{b_{c x}}\right)\left(\frac{1}{f_{y t}}\right)}, \\
& \rho_{s y}=\sum A_{s y} f_{y t} \sqrt{\left(\frac{b_{c x}}{s}\right)\left(\frac{b_{c x}}{b_{c y}}\right)\left(\frac{1}{f_{y t}}\right) .}
\end{aligned}
$$

3.4. The Constitutive Equations of Connecting Steels considering Shear Stress Influence. The dowel action shear stress caused by joint interface slip will reduce the yield strength of the connecting steels. The effect of shear stress due to bending curvature (10) on the yield stress of the bar can be taken into consideration by applying the Von Mises yield criterion. The reduced steel stress-strain constitutive equations are as follows and the curves are in Figure 10.

Elastic stage is as follows:

$$
\sigma=E_{s} \varepsilon, \quad\left(\sigma<f_{y c}^{\prime}\right) .
$$

Yield stage is as follows:

$$
\sigma=f_{y c}^{\prime}=\sqrt{f_{y}^{\prime}-\frac{\left(V-\mu_{f} N\right)}{A_{s}^{\prime}} .}
$$

3.5. The Moment Calculation Equations for Precast Shear Wall. For the value of $V$ in (27), it should be determined by (17), considering that the shear capacity of the precast joint should be not less than that of the whole shear wall. So this $V$ value is larger than the actual shear force meaning that the yield strength of the steel bars is lower than the actual strength and thusly the design results will be safer.

Using $f_{y c}^{\prime}$ to replace $f_{y}^{\prime}$ in (14),

$$
\begin{aligned}
N_{f} \leq & \alpha_{1} f_{c}\left[\xi b h_{0}+\left(b_{f}^{\prime}-b\right) h_{f}^{\prime}\right]+f_{y c}^{\prime} A_{s}^{\prime}-\sigma_{s} A_{s} \\
& +\left(1+\frac{\xi-\beta_{1}}{0.5 \beta_{1} \omega}\right) f_{y c}^{\prime} A_{s w} .
\end{aligned}
$$

By solving (28), the relative compression region height $\xi$ is as follows:

$$
\begin{aligned}
\xi= & \frac{0.5 \beta_{1} \omega N_{c}+\beta_{1} f_{y c}^{\prime} A_{s w}}{0.5 \beta_{1} \alpha_{1} f_{c} b h_{w}+f_{y c}^{\prime} A_{s w}}, \\
N_{c}= & N_{f}-\alpha_{1} f_{c}\left(b_{f}^{\prime}-b\right) h_{f}^{\prime}-f_{y c}^{\prime} A_{s}^{\prime}+\sigma_{s} A_{s} \\
& -f_{y c}^{\prime} A_{s w} .
\end{aligned}
$$


TABLE 1: Calculated and experimental tested results $(\mathrm{kN})$.

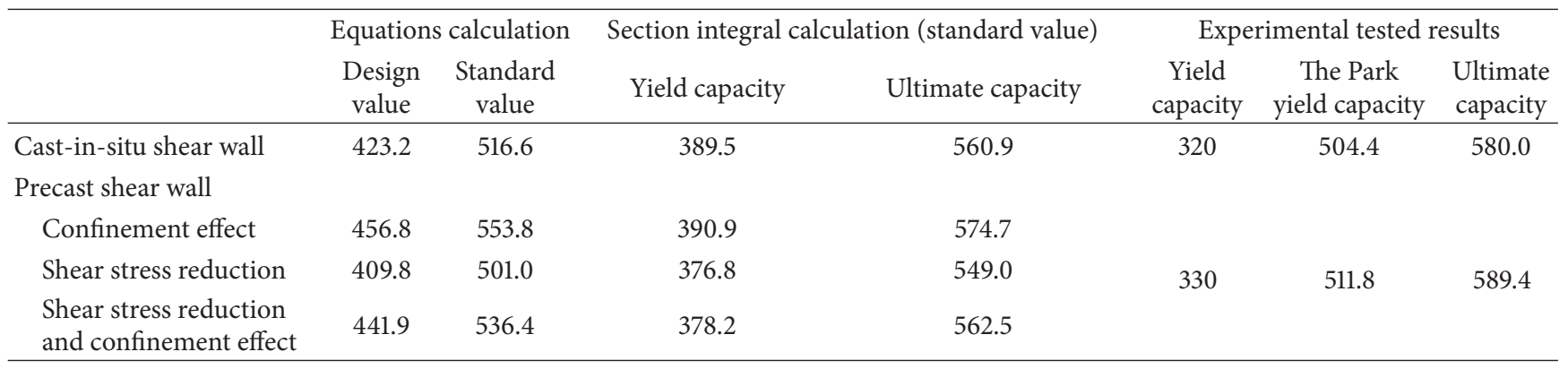

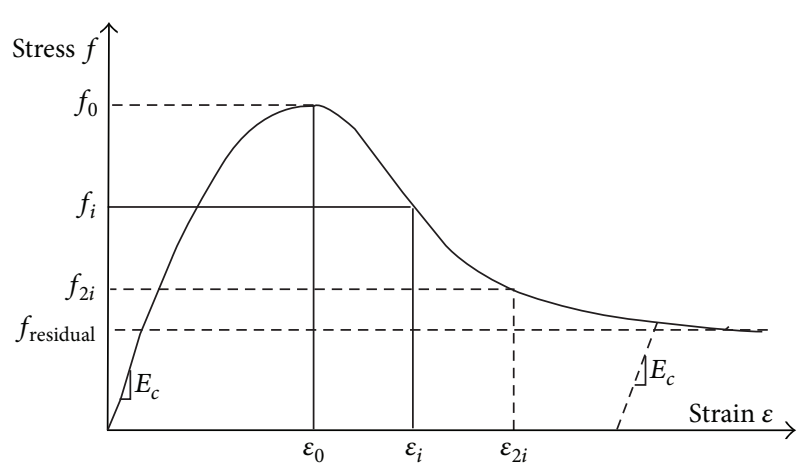

FIGURE 9: The stress-strain curve of confined concrete.

Taking $\xi$ into (15), the eccentric distance can be obtained:

$$
\begin{aligned}
e & =\frac{\alpha_{1} f_{c} I_{c w}+f_{y}^{\prime} A_{s}^{\prime}\left(h_{0}-a_{s}^{\prime}\right)+M_{s w}}{N_{f}}, \\
I_{c w} & =\xi(1-0.5 \xi) b h_{0}^{2}+\left(b_{f}^{\prime}-b\right) h_{f}^{\prime}\left(h_{0}-\frac{h_{f}^{\prime}}{2}\right) .
\end{aligned}
$$

And then, using (29) and (31), the moment strength will be obtained (33). Use $f_{0}$ obtained from (21) to replace $f_{c}$ in (33) and the improvement of the confined concrete will be considered:

$$
\begin{aligned}
& M_{f} \\
& =\alpha_{1} f_{c}\left[\xi(1-0.5 \xi) b h_{0}^{2}+\left(b_{f}^{\prime}-b\right) h_{f}^{\prime}\left(h_{0}-\frac{h_{f}^{\prime}}{2}\right)\right] \\
& \quad+f_{c y}^{\prime} A_{s}^{\prime}\left(h_{0}-a_{s}^{\prime}\right)+M_{s w}+N_{f}\left(a-\frac{h}{2}-e_{a}\right) .
\end{aligned}
$$

\section{Verification on the Capacity Calculation Equations for Precast Grouted Shear Wall}

Using the equations obtained above and the section fiber stress integral approach, one example was calculated to verify the accuracy of the capacity calculation approach for precast grouted shear wall. Apart from the numerical calculations, one cast-in-situ specimen and two precast shear

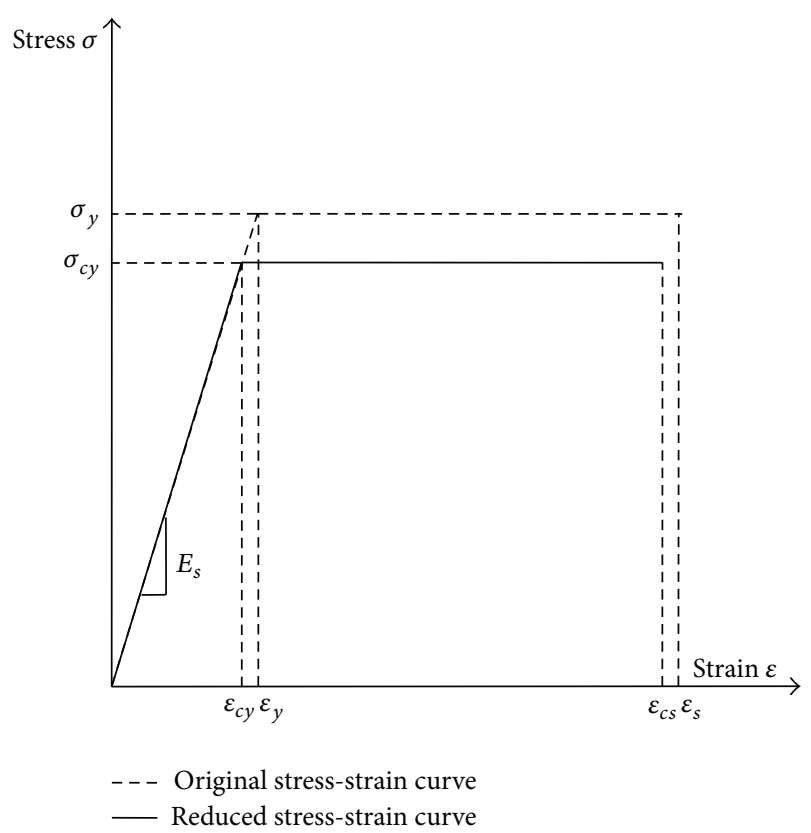

FIGURE 10: The reduced steel stress-strain constitutive curve.

wall specimens, having the same size and parameter with the calculated example, were tested. The specimen size and parameter are shown in Figure 9. The calculated results and the experimental tested results are presented in Table 1 . The calculated cast-in-situ capacities and the experimental tested capacities of cast-in-situ specimen are close, while the calculated capacities are little lower than that of the experimental specimen (Figure 11). After considering the reduction of the precast joint interface slip, the calculated capacities of precast specimens exhibit a further reduction than that of cast-in-situ specimen, which indicates that the equations can reflect the reduction of the precast joint to the properties of precast shear wall. All the calculated results approved that the capacity calculation approach can accurately and reasonably suit for the precast shear wall capacity calculation and the calculated results reflect a certain safety capacity.

The results in Table 1 also illustrated that when considering the confined concrete improvement, the calculated capacity increased. When considering both the confined concrete improvement and the joint interface slip reduction, the calculated precast shear wall capacities are lower 


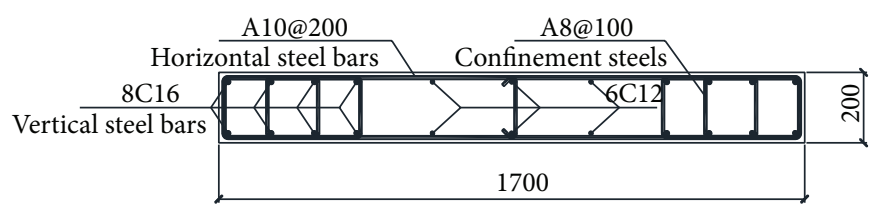

(a) The cross section of cast-in-situ specimen

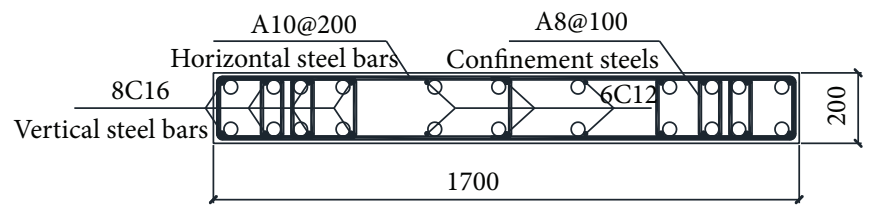

(c) The cross section of precast specimens

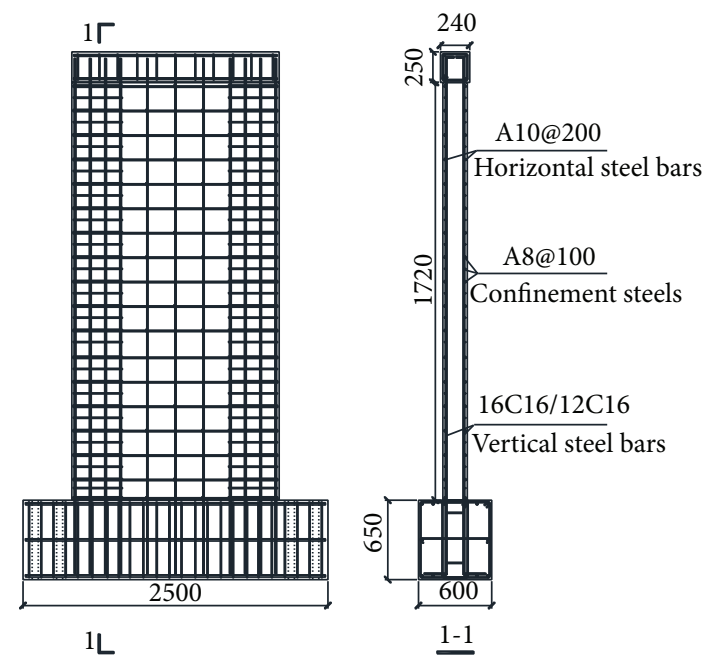

(b) The steel arrangement of cast-in-situ specimen

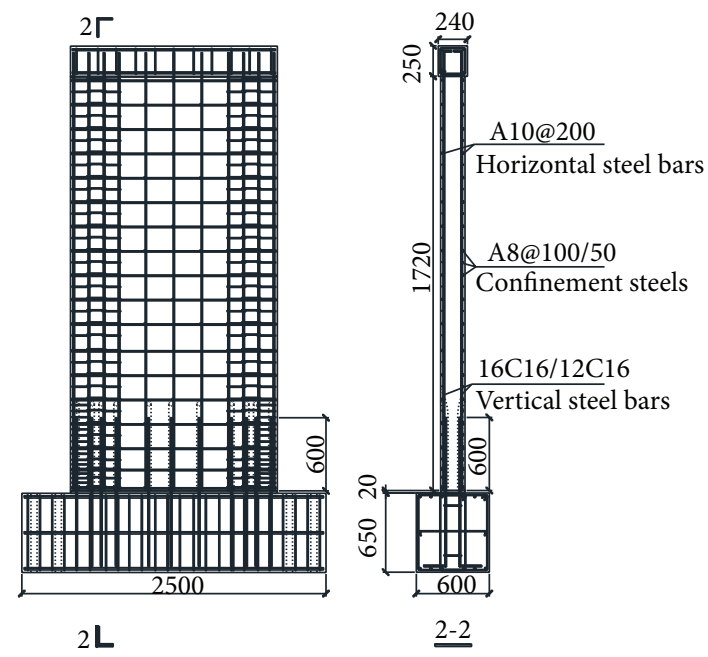

(d) The steel arrangement of specimens

FIGURE 11: The specimen size and steel configuration (mm).

than that only considering the improvement while they are higher than that only considering the reduction. This phenomenon indicated that even though the precast joint interface slip reduced the yield strength of connecting steel bars, this reduction can be completely eliminated by utilizing sufficient confinement steels to improve the concrete properties.

But in some cases, especially for those precast structures in high seismic areas, a higher safety capacity will be required. So, this paper suggests that, for the normal precast structures in low or medium seismic area, the capacity calculation approach considering both the reduction of interface slip and the improvement of confinement steels can be applied in the design of precast shear wall. While for both the normal precast structures in high seismic areas, and the special and high-rise structures in medium seismic areas, in order to have a higher safety capacity, the capacity calculation approach should only consider the reduction of interface slip.

\section{Conclusions}

Gap opening displacement and joint interface slip in the precast joint hardly can be avoided and will cause dramatic influences on the connecting steels' mechanical properties. The increasing of gap opening displacement will reduce the dowel action shear stress while the increasing of joint interface slip will increase in a certain range and then decline the dowel action shear stress. But the gap opening displacement influence is more dramatic than the joint interface slip. As a result, the shear stress carried by the tension connecting steel bars will be low enough to be ignored due to the large gap opening displacement influence. Therefore, only the compression steel bars' yield strength should be reduced considering the dowel action shear stress effect (Equation (30)).

Based on the properties of precast joint, a simplified precast joint section calculation model was proposed. Considering the influence of joint interface slip and excessive 
confinement steels, the eccentric compression or eccentric tension strength calculating equations for cast-in-situ shear wall were verified to suit for precast shear wall. The comparison between calculated results and experimental tested results of example of shear walls indicated that this capacity calculation approach is accurate and reasonable for precast shear wall.

From the calculated results of precast shear wall, this paper suggests that the calculation approach should consider both the interface displacements reduction and the confinement steels improvement in the design of normal precast structures in low or medium seismic area. While when design both the normal precast structures in high seismic areas and the special and high-rise precast structures in medium seismic areas, in order to achieve a higher safety capacity, this calculation approach should only consider the steel yield strength reduction and the confinement steels improvement should be ignored.

\section{Nomenclature}

A: Sectional area of shear wall

$A_{s}$ : Sectional area of connecting steels in precast joint compression region

$A_{\text {sh }}$ : Sectional area of a single horizontal steel bar section

$A_{s x}$ : Sectional area of rectangular confinement steels in $x$ direction

$A_{s y}$ : Sectional areas of rectangular confinement steels in $y$ direction

$A_{w}$ : Sectional area of web portion for $\mathrm{T}$ and $\mathrm{I}$ shape shear wall

$A_{s}^{\prime}$ : Sectional area of the steel bars in compression region

$b_{c x}$ : Loop width of rectangular confinement steels in $x$ direction

$b_{c y}$ : Loop width of rectangular confinement steels in $y$ direction

$d_{s}: \quad$ Diameter of steel bar

$E_{c}$ : Secant modulus of concrete

$E_{s}$ : Elastic modulus of steel bar

$E_{t i}$ : Initial tangent modulus at zero stress of confined concrete

$e_{a}$ : Appending of eccentricity distance

$f_{c k}$ : Uniaxial compressive strength of concrete

$f_{t k}$ : Tensile strength of concrete

$f_{y t}$ : Yield strength of confinement steel bar

$f_{y w}$ : Yield strength of uniform configured vertical steel bars

$f_{y}^{\prime}$ : Initial yield strength of steel bars

$f_{y c}^{\prime}$ : Reduced yield strength of steel bars

$h_{0}$ : Whole sectional height of shear wall

$I_{s}$ : Sectional inertia moment of steel bar

$M$ : Total moment acting on shear wall forward section

$M_{f}$ : Design moment capacity of shear wall forward section
$M_{s w}$ : Moment capacity of uniform configured vertical steel bars to the center of margin tension steels

N: Total axial force acting on precast shear wall

$N_{f}$ : Design axial force capacity of shear wall forward section

$N_{s w}$ : Axial compression capacity of uniform configured vertical steel bars

$P$ : Axial tension load on steel bar

$s$ : Vertical distance of confinement steel loops

$s_{v}: \quad$ Vertical distance of horizontal steel bars

$V: \quad$ Total lateral shear force acting on precast shear wall

$V_{f}$ : Design shear force capacity of shear wall forward section

$\delta_{b}$ : Normal direction slip of steel bar which equals half the joint interface slip

$\delta_{v}: \quad$ Axial slip of spliced steel bar which equals the gap opening displacement

$\varepsilon_{c}: \quad$ Strain at the maximum stress of concrete under uniaxial compression

$\lambda$ : Shear span ratio of calculated critical section

$\mu_{f}:$ Frictional ratio between concrete surfaces

$\omega$ : Distance ratio of uniform steel bars portion's height to whole section's height $h_{0}$.

\section{Conflict of Interests}

The authors declare that there is no conflict of interests regarding the publication of this paper.

\section{Acknowledgment}

Financial support from the National Science \& Technology Pillar Program during the Twelfth Five-Year Plan Period of China under Grant no. 2011BAJ10B03 is acknowledged.

\section{References}

[1] M. Kramar, T. Isaković, and M. Fischinger, "Seismic collapse risk of precast industrial buildings with strong connections," Earthquake Engineering and Structural Dynamics, vol. 39, no. 8, pp. 847-868, 2010.

[2] B. J. Smith, Y. C. Kurama, and M. J. McGinnis, "Behavior of precast concrete shear walls for seismic regions: comparison of hybrid and emulative specimens," Journal of Structural Engineering, vol. 139, no. 11, pp. 1917-1927, 2013.

[3] S. El-Tawil, K. A. Harries, P. J. Fortney, B. M. Shahrooz, and Y. Kurama, "Seismic design of hybrid coupled wall systems: state of the art," Journal of Structural Engineering, vol. 136, no. 7, pp. 755-769, 2010.

[4] N. M. Hawkins and S. K. Ghosh, "Requirements for the use of PRESSS moment-resisting frame systems," PCI Journal, vol. 49, no. 2, pp. 98-103, 2004.

[5] A. A. Yee, "Social and environmental benefits of precast concrete technology," PCI Journal, vol. 46, no. 3, pp. 14-19, 2001. 
[6] A. A. Sayadi, A. B. A. Rahman, M. Z. B. Jumaat, U. Johnson Alengaram, and S. Ahmad, "The relationship between interlocking mechanism and bond strength in elastic and inelastic segment of splice sleeve," Construction and Building Materials, vol. 55, pp. 227-237, 2014.

[7] L. Jiabin, G. Zhengxing, and C. Leqi, "Research on precast roof truss of prestressed concrete square pile," in Proceedings of the International Conference on Advanced Engineering Materials and Architecture Science, Applied Mechanics and Materials, pp. 359-364, Xian, China, 2014.

[8] S.-M. Kang, O.-J. Kim, and H.-G. Park, "Cyclic loading test for emulative precast concrete walls with partially reduced rebar section," Engineering Structures, vol. 56, pp. 1645-1657, 2013.

[9] M. Soltani, X. An, and K. Maekawa, "Localized nonlinearity and size-dependent mechanics of in-plane RC element in shear," Engineering Structures, vol. 27, no. 6, pp. 891-908, 2005.

[10] J. H. Ling, A. B. Abd. Rahman, I. S. Ibrahim, and Z. Abdul Hamid, "Behaviour of grouted pipe splice under incremental tensile load," Construction and Building Materials, vol. 33, no. 8, pp. 90-98, 2012.

[11] GB 50011-2010, Code for Seismic Design of Buildings, China Architecture \& Building Press, Beijing, China, 2010.

[12] ACI Committee 318, Building Code Requirements for Structural Concrete (ACT 318-11), American Concrete Institute, Farmington Hills, Mich, USA, 2011.

[13] Z.-C. Deng and J.-L. Qu, "The experimental studies on behavior of ultrahigh-performance concrete confined by hybrid fiberreinforced polymer tubes," Advances in Materials Science and Engineering, vol. 2015, Article ID 201289, 18 pages, 2015.

[14] A. K. Samani and M. M. Attard, "A stress-strain model for uniaxial and confined concrete under compression," Engineering Structures, vol. 41, pp. 335-349, 2012. 

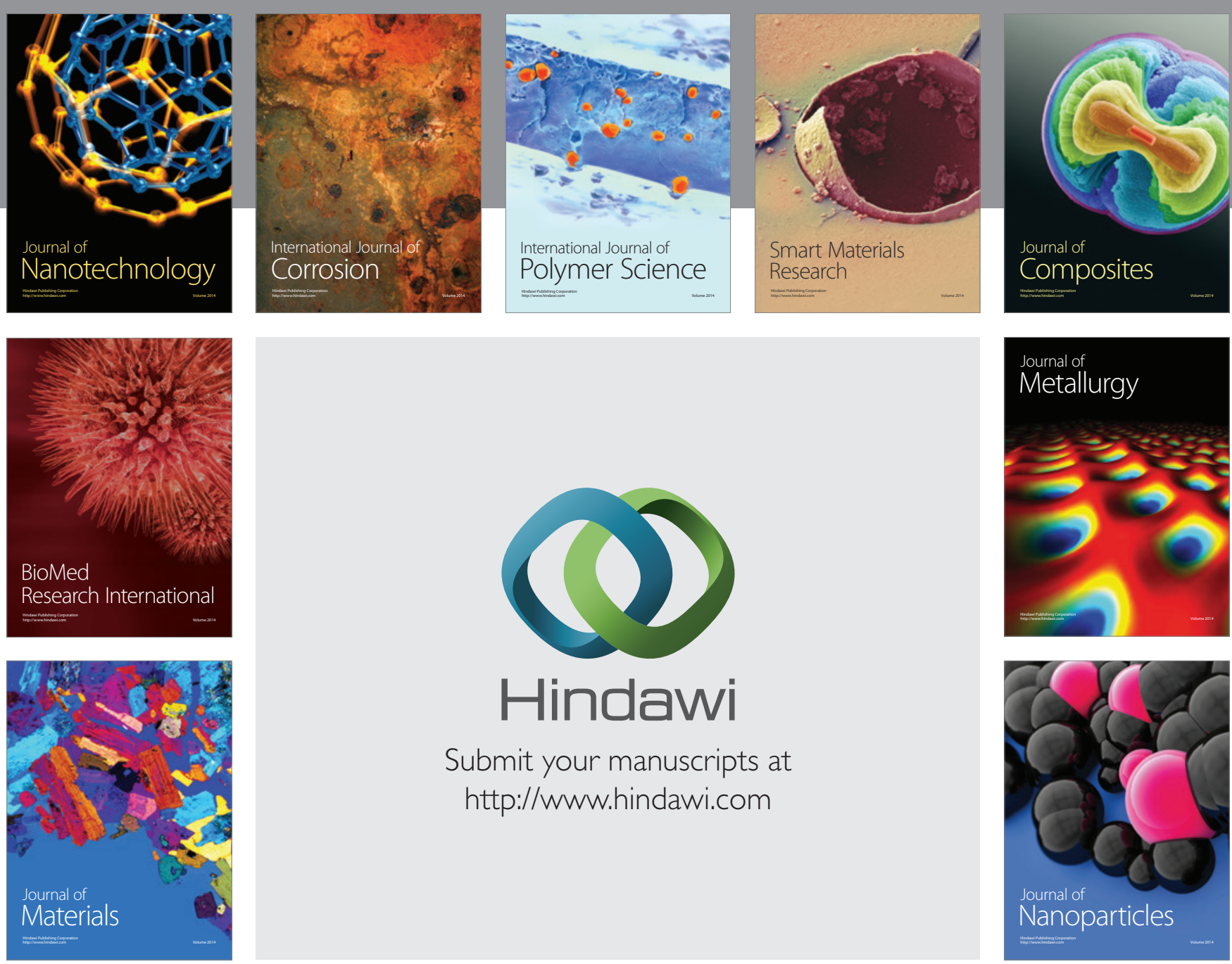

Submit your manuscripts at http://www.hindawi.com
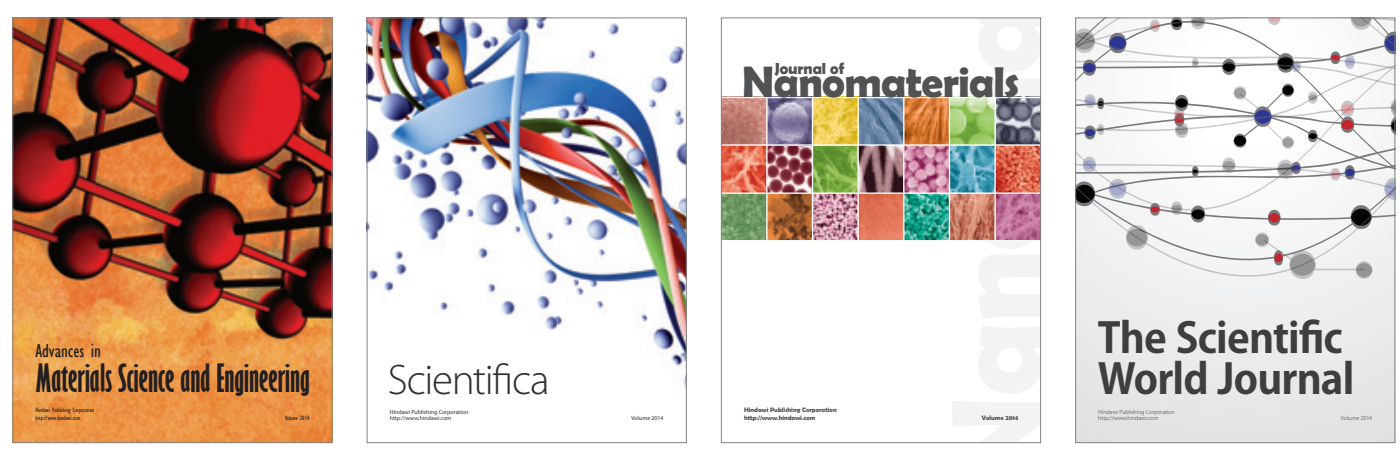

\section{The Scientific World Journal}
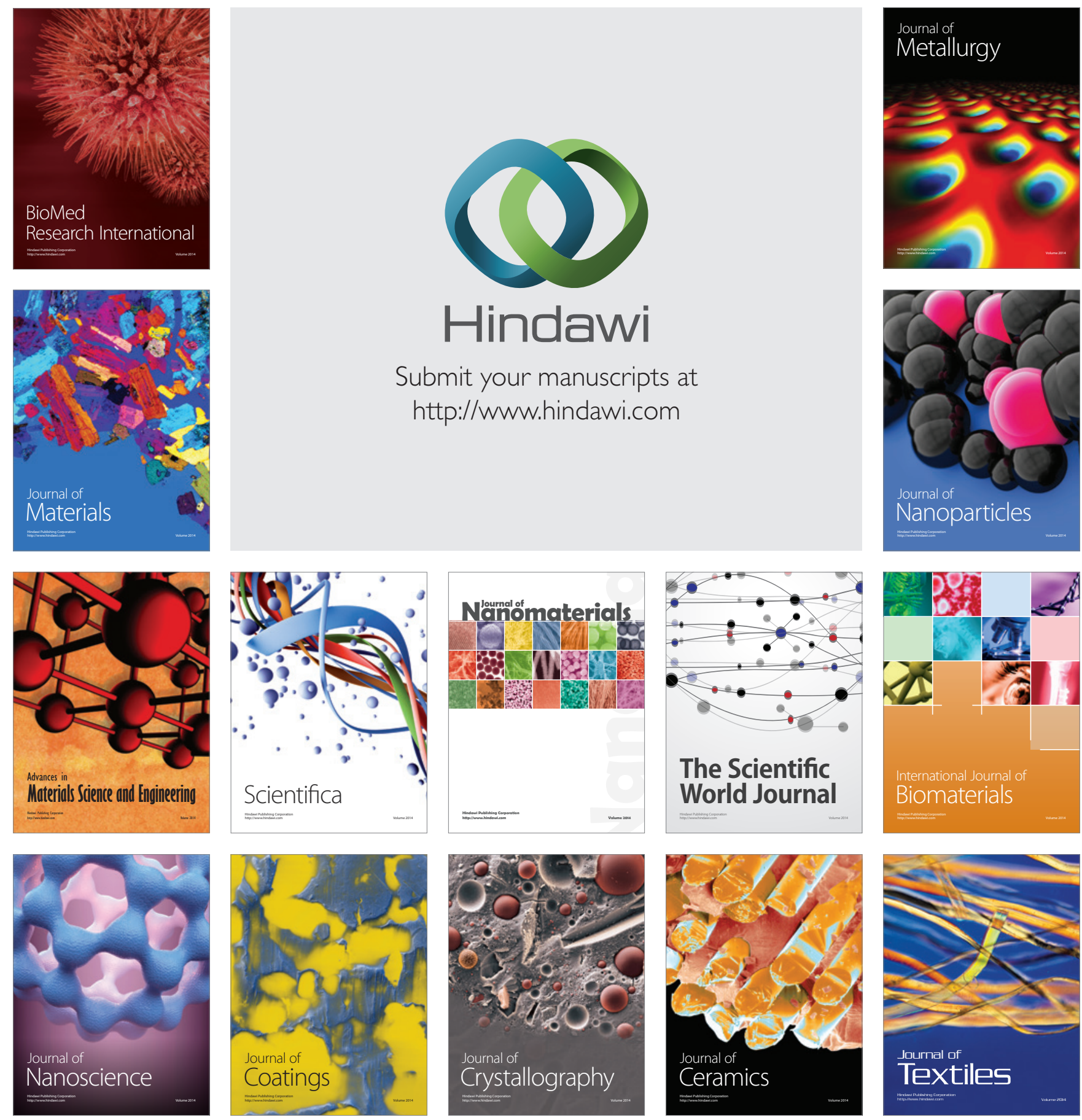